\title{
The Loewner equation and Lipschitz graphs
}

\author{
Steffen Rohde, Huy Tran and Michel Zinsmeister
}

\begin{abstract}
The proofs of continuity of Loewner traces in the stochastic and in the deterministic settings employ different techniques. In the former setting of the Schramm-Loewner evolution SLE, Hölder continuity of the conformal maps is shown by estimating the derivatives, whereas the latter setting uses the theory of quasiconformal maps. In this note, we adopt the former method to the deterministic setting and obtain a new and elementary proof that Hölder-1/2 driving functions with norm less than 4 generate simple arcs. We also give a sufficient condition for driving functions to generate curves that are graphs of Lipschitz functions.
\end{abstract}

\section{Introduction and results}

The chordal Loewner differential equation

$$
\partial_{t} g_{t}(z)=\frac{2}{g_{t}(z)-\lambda_{t}}, \quad g_{0}(z)=z,
$$

which will be briefly reviewed in Section 2, provides a one-to-one correspondence between certain decreasing families of simply connected subdomains $\mathbb{H} \backslash K_{t}$ of the upper half plane, and real-valued continuous functions $\lambda_{t}$. Initially developed as a tool to study extremal problems in complex analysis [7], it has become an important tool in probability theory, based on Oded Schramm's insight [11] that Brownian motion arises naturally as the driving function $\lambda_{t}$ in various settings of random sets $K_{t}$.

While it is not hard to see that for simple curves $\gamma \subset \mathbb{H} \cup\{0\}$ with $\gamma_{0}=0$, the family $K_{t}=\gamma[0, t]$ yields continuous functions $\lambda_{t}$ (see Section 2 for a precise statement), the converse is not true in general: There are continuous functions $\lambda_{t}$ for which the associated hulls $K_{t}$ are not locally connected, and hence not of the form $\gamma[0, t]$ for some continuous function $\gamma$.

In [8] and [6], the continuity problem was treated by viewing the hulls $K_{t}$ as the result of "conformally welding" two intervals of the real line, and by applying the theory of quasiconformal maps to the welding problem. The main result is:

Mathematics Subject Classification (2010): Primary 30C20; Secondary 34M10.

Keywords: Loewner differential equation. 
Theorem 1.1 ([8], [6]). If the driving function $\lambda$ has Hölder-1/2 norm less than 4, then the chordal Loewner equation generates a simple curve $\gamma$. Moreover, $\gamma$ is a quasiconformal arc that meets the real line non-tangentially.

The constant 4 in the theorem is sharp in the sense that for each $c \geq 4$, there exists a driving function whose Hölder-1/2 norm is $c$ and the Loewner equation generates a curve that meets the real line other than at the base, see examples in $[3]$ and $[6]$.

In the stochastic setting of the Schramm-Loewner evolution $\mathrm{SLE}_{\kappa}$ where the driving term is $\lambda_{t}=\sqrt{\kappa} B_{t}$ and $B_{t}$ is a standard one-dimensional Brownian motion, the conformal welding approach leads to interesting and difficult problems, see [12] and [1] for related deep results. In [10], the almost sure continuity of the $\mathrm{SLE}_{\kappa}$ hulls was proved by different means: Based on estimates of the expectation $E\left[\left|\left(g_{t}^{-1}\right)^{\prime}(z)\right|^{p}\right]$ for suitable exponents $p$, it was shown that $\lim _{y \rightarrow 0} g_{t}^{-1}\left(\lambda_{t}+i y\right)$ exists, is continuous in $t$, and that this implies continuity of the hulls.

In this note, we will adopt the second method to the deterministic setting, and obtain a new and elementary proof of Theorem 1.1. The key observation is the following (see Theorem 3.1 below): under the "upward" flow (2.3), the point $z_{t}=f_{t}(i)-\lambda_{t}$ will never leave the cone $\{|x|<c y\}$, where $c$ depends on the Hölder-1/2 norm only. Combined with the integral representation of $\log \left|f_{t}^{\prime}(z)\right|$ ((2.8) below), this easily gives an estimate for $\left|\left(g_{t}^{-1}\right)^{\prime}\left(\lambda_{t}+i y\right)\right|$ that, when integrated, implies existence and continuity of $\lim _{y \rightarrow 0} g_{t}^{-1}\left(\lambda_{t}+i y\right)$. Standard conformal mapping techniques (particularly the Gehring-Hayman inequality) then yield the additional information that the trace is a quasiconformal arc approaching the real line non-tangentially. When applied to the integral representation $(2.9)$ of $\arg f_{t}^{\prime}(z)$, our approach also gives a sufficient condition for the driving functions to generate the graph of a Lipschitz function:

Theorem 1.2. There exists a constant $C_{0}>0$ such that, for every continuous function $\lambda$ satisfying

$$
\int_{0}^{t} \frac{N_{s, t}^{\lambda}}{(t-s)^{3 / 2}} d s \leq C_{0} \quad \text { for all } 0<t<T,
$$

where $N_{s, t}^{\lambda}=\sup \left\{\left|\lambda_{r}-\lambda_{s}\right|: s \leq r \leq t\right\}$, the Loewner equation generates a graph of a Lipschitz function.

Theorem 1.2 is sharp, as the example $\lambda_{t}=c \sqrt{1-t}(0 \leq t \leq 1)$ shows (see [3]). In this example, the trace is asymptotic to a logarithmic spiral at the tip, hence it is not the graph of any function, and the integral in Theorem 1.2 diverges. On the other hand, the straight line of angle $\pi \alpha$ has driving function $\lambda_{t}=c \sqrt{t}$, where $c=2(1-2 \alpha) / \sqrt{\alpha(1-\alpha)}$, so that every Hölder-1/2 norm can arise from a simple curve. Notice that for $\lambda_{t}=c \sqrt{t}$, the integral has the finite value $c \sqrt{2} \sinh ^{-1}(1)$.

The organization of the paper is as follows. In Section 2 we briefly review basic facts and definitions around the Loewner equation. The key points lie in Section 3, where we state and prove Theorem 3.1 regarding the trajectories $z_{t}=f_{t}(i t)-\lambda_{t}$ of the backward Loewner flow when the Hölder-1/2 norm is less than 4. Finally, Theorems 1.1 and 1.2 will be proved in Section 4 . 


\section{Preliminaries}

Let $\gamma:[0, T] \rightarrow \mathbb{H} \cup\{0\}$ be a simple curve in the upper half-plane $\mathbb{H}$ except that $\gamma_{0} \in \mathbb{R}$. For each $t \in[0, T]$, by the Riemann mapping theorem there exists a unique conformal map $g_{t}: \mathbb{H} \backslash \gamma[0, t] \rightarrow \mathbb{H}$ satisfying the hydrodynamic normalization:

$$
g_{t}(z)=z+\frac{c_{t}}{z}+\cdots \quad \text { when } z \rightarrow \infty
$$

It can be shown that $c_{t}$ is a nonnegative, strictly increasing, continuous function and $c_{0}=0$ [4]. Hence one can reparametrize $\gamma$ so that $c_{t}=2 t$. Then for each $z \in \mathbb{H}$, the function $t \mapsto g_{t}(z)$ satisfies the (downward) chordal Loewner equation:

$$
\partial_{t} g_{t}(z)=\frac{2}{g_{t}(z)-\lambda_{t}}, \quad g_{0}(z)=z
$$

where $\lambda$ is a continuous, real-valued function and $g_{t}\left(\gamma_{t}\right)=\lambda_{t}$, see Chapter 4 in [4].

Conversely, if one starts with a continuous function $\lambda_{t}:[0, T] \rightarrow \mathbb{R}$, one can consider the initial value problem for each $z \in \mathbb{H}$ :

$$
\partial_{t} g(t, z)=\frac{2}{g(t, z)-\lambda_{t}}, \quad g(0, z)=z .
$$

For each $z \in \mathbb{H}$ there is a maximal interval for which a solution $g(t, z)$ exists. Let $T_{z}=\sup \{s \in[0, T]: g(t, z)$ exists on $[0, s)\}$. It is easy to see that, if $T_{z}<T$, then

$$
\lim _{t \rightarrow T_{z}} g(t, z)=\lambda_{T_{z}}
$$

Let $H_{t}=\left\{z \in \mathbb{H}: T_{z}>t\right\}$ and $g_{t}(z)=g(t, z)$. Then one can show that the set $H_{t}$ is a simply connected subdomain of $\mathbb{H}$, and $g_{t}(z)$ is the unique conformal map from $H_{t}$ onto $\mathbb{H}$ with the following normalization near infinity:

$$
g_{t}(z)=z+\frac{2 t}{z}+O\left(\frac{1}{z^{2}}\right)
$$

The driving function $\lambda$ of the Loewner chain $\left(g_{t}\right)$ is said to generate a curve if there exists a curve $\gamma$ such that $H_{t}$ is the unbounded component of $\mathbb{H} \backslash \gamma[0, t]$ for each $t \geq 0$. By Theorem 4.1 in [10], this is equivalent to the existence and continuity in $t>0$ of

$$
\gamma_{t}:=\lim _{y \rightarrow 0+} g_{t}^{-1}\left(\lambda_{t}+i y\right)
$$

By Proposition 2.19 of [5] and Proposition 3.11 of [2], a very useful and simple criterion for this existence and continuity is the convergence to zero of

$$
v(t, \varepsilon):=\int_{0}^{\varepsilon}\left|\left(g_{t}^{-1}\right)^{\prime}\left(\lambda_{t}+i y\right)\right| d y
$$

as $\varepsilon \rightarrow 0$, uniformly in $t \in[0, T]$. 
Rather than directly working with the Loewner equation (2.1), it is often easier to work with the upward Loewner equation:

$$
\partial_{t} f_{t}(z)=\frac{-2}{f_{t}(z)-\xi_{t}}, \quad f_{0}(z)=z,
$$

for $z \in \mathbb{H}$ and real-valued continuous function $\xi_{t}$. Since the imaginary part of $f_{t}(z)$ is strictly increasing, the solution exists for all time $t \geq 0$. If $\left(g_{t}\right)_{0 \leq t \leq T}$ is the solution to (2.1) with driving function $\lambda$ and if $\left(f_{t}\right)_{0 \leq t \leq T}$ is the solution to $(2.3)$ with $\xi_{t}=\lambda_{T-t}$, then

$$
f_{T}(z)=g_{T}^{-1}(z)
$$

We will frequently use the following two simple properties of the Loewner equation, regarding the translation and concatenation of driving functions:

First, if $a \in \mathbb{R}$ and $\tilde{\xi}_{t}=\xi_{t}+a$, then the Loewner chain $\left(\tilde{f}_{t}\right)$ corresponding to $\tilde{\xi}$ is given by

$$
\tilde{f}_{t}(z)=f_{t}(z-a)+a .
$$

Second, let $\left(f_{t}^{(1)}\right)_{0 \leq t \leq T_{1}}$ (respectively $\left(f_{t}^{(2)}\right)_{0 \leq t \leq T_{2}}$ ) be the solution to (2.3) with the driving function $\xi^{(\overline{1})}$ defined on $\left[0, T_{1}\right]$ (respectively $\xi^{(2)}$ defined on $\left[0, T_{2}\right]$ ). Suppose $\xi_{T_{1}}^{(1)}=\xi_{0}^{(2)}$, and define the concatenation of $\xi^{(1)}$ and $\xi^{(2)}$ by

$$
\xi_{t}=\left\{\begin{array}{cc}
\xi_{t}^{(1)}, & t \in\left[0, T_{1}\right], \\
\xi_{t-T_{1}}^{(2)}, & t \in\left[T_{1}, T_{1}+T_{2}\right] .
\end{array}\right.
$$

Then the (upward) Loewner solution corresponding to $\xi$ is given by

$$
f_{t}=\left\{\begin{array}{cc}
f_{t}^{(1)}, & t \in\left[0, T_{1}\right], \\
f_{t-T_{1}}^{(2)} \circ f_{T_{1}}^{(1)}, & t \in\left[T_{1}, T_{1}+T_{2}\right] .
\end{array}\right.
$$

The following notation will be used throughout the rest of the paper: if $z \in \mathbb{H}$ and $f_{t}(z)$ is the solution to $(2.3)$, we define $x_{t}:=x_{t}(z, \xi)$ and $y_{t}:=y_{t}(z, \xi)$ by

$$
x_{t}+i y_{t}:=z_{t}:=f_{t}(z)-\xi_{t} .
$$

It follows that

$$
\partial_{t}\left(x_{t}+\xi_{t}\right)=\frac{-2 x_{t}}{x_{t}^{2}+y_{t}^{2}}
$$

and

$$
\partial_{t} y_{t}=\frac{2 y_{t}}{x_{t}^{2}+y_{t}^{2}}
$$

The following expressions for $\left|f_{t}^{\prime}(z)\right|$ and $\arg f_{t}^{\prime}(z)$ in terms of $x_{t}$ and $y_{t}$ will be used to prove Theorems 1.1 and 1.2. Since

$$
f_{t}^{\prime}(z)=e^{\log f_{t}^{\prime}(z)}=e^{\int_{0}^{t} \partial_{s} \log f_{s}^{\prime}(z) d s}
$$

and

$$
\partial_{s} \log f_{s}^{\prime}(z)=\frac{\partial_{s} f_{s}^{\prime}(z)}{f_{s}^{\prime}(z)}=\frac{2}{\left(f_{s}(z)-\xi(s)\right)^{2}},
$$


we have

$$
\left|f_{t}^{\prime}(z)\right|=\exp \left(2 \int_{0}^{t} \frac{x_{s}^{2}-y_{s}^{2}}{\left(x_{s}^{2}+y_{s}^{2}\right)^{2}} d s\right)=\exp \left(\int_{0}^{t} \frac{x_{s}^{2}-y_{s}^{2}}{x_{s}^{2}+y_{s}^{2}} \cdot \frac{2 d s}{x_{s}^{2}+y_{s}^{2}}\right)
$$

and

$$
\arg f_{t}^{\prime}(z)=-4 \int_{0}^{t} \frac{x_{s} y_{s}}{\left(x_{s}^{2}+y_{s}^{2}\right)^{2}} d s .
$$

Finally, we will frequently use the following simple estimate for the oscillation of $x_{t}$ for general driving functions.

Lemma 2.1. Let $\xi$ be an arbitrary continuous function.

a) If $x_{s} \geq 0$ for all $0 \leq s \leq t$, then $x_{t} \leq x_{0}+\xi_{0}-\xi_{t}$.

b) In general, $\left|x_{t}\right| \leq\left|x_{0}\right|+M_{0, t}^{\xi}$, where $M_{0, t}^{\xi}=\sup \left\{\left|\xi_{r}-\xi_{t}\right|: r \in[0, t]\right\}$.

Proof. Since $x_{s} \geq 0$, the sum $x_{t}+\xi_{t}$ is nonincreasing by (2.6), and part a) follows. To prove b), by symmetry we may assume that $x_{0} \geq 0$, and we may also assume $\left|x_{t}\right|>x_{0}$, else b) is trivial. Let $S=\sup \left\{0 \leq s<t:\left|x_{s}\right| \leq x_{0}\right\}$ so that $\left|x_{S}\right|=x_{0}$.

If $x_{t} \geq 0$ then $x_{t}>x_{0}$ and $x_{0}=x_{S}<x_{s}$ for $S<s \leq t$. Applying a) with $z$ replaced by $x_{S}+i y_{S}$ and $\xi$ replaced by $\xi(\cdot+S)$ we get

$$
x_{t} \leq x_{S}+\xi_{S}-\xi_{t}=x_{0}+\xi_{S}-\xi_{t} .
$$

If $x_{t}<0$ then $x_{t}<-x_{0}$ and $x_{s}<x_{S}=-x_{0}$ for $S<s \leq t$. Now replacing $z$ by $-\left(x_{S}+i y_{S}\right)$ and $\xi$ by $-\xi \cdot+S$, the claim follows again from a).

\section{Staying in a fixed cone}

In this section, we restrict our attention to the upward Loewner equation (2.3) with driving function $\xi$ whose Hölder-1/2 norm satisfies

$$
\sigma:=\|\xi\|_{1 / 2}=\sup _{s \neq t} \frac{\left|\xi_{t}-\xi_{s}\right|}{|t-s|^{1 / 2}}<4 .
$$

Denote $A_{c}$ the cone $\{x+i y:|x| \leq c y\}$ and $A_{c}(v)=v+A_{c}$ for $v \in \mathbb{R}$. The main result of this section is:

Theorem 3.1. There is a constant $c_{\sigma}$ such that, if $z_{0}=i y$, then $z_{t}=f_{t}\left(z_{0}+\xi_{0}\right)-\xi_{t}$ stays in the cone $A_{c_{\sigma}}$ for all $t$. Moreover,

$$
\sqrt{\frac{4 t}{1+c_{\sigma}^{2}}+y^{2}} \leq y_{t} \leq \sqrt{4 t+y^{2}}
$$

for all $t \geq 0$, and $c_{\sigma} \leq \sigma / \sqrt{4-\sigma^{2}}$ for $\sigma<2$.

This theorem easily implies the Hölder continuity of $f_{t}$, Corollary 3.5 below. The intuition behind the proof of Theorem 3.1 is as follows. To first order, $\Delta z_{t}=$ $\frac{-2}{z_{t}} \Delta t-\Delta \xi$. Therefore, the larger $x_{t} / y_{t}$, the stronger $\frac{2}{z_{t}} \Delta t$ pushes towards the middle of the cone, and dominates $\Delta \xi$ if the Hölder- $1 / 2$ norm is small. 
We will first show that an upper bound on the growth rate of $x_{t}$ implies a lower bound on $y_{t}$ that is comparable to the optimal upper bound $y_{t} \leq \sqrt{4 t+y_{0}^{2}}$.

Lemma 3.2. If $\left|x_{t}\right|<M \sqrt{t}$ for all $t \geq C y_{0}^{2}$ with some $M<2$ and $C>0$, then

$$
y_{t}^{2} \geq L t
$$

for all $t \geq 0$, where $L=\min \left(1 / C, 4-M^{2}\right)>0$.

Proof. Since $L \leq 1 / C$, we have $L t \leq t / C \leq y_{0}^{2}<y_{t}^{2}$ for $0<t \leq t_{0}:=C y_{0}^{2}$, where the last inequality comes from the fact that $y_{t}$ is increasing in $t$ which can be seen from the equation (2.7). If (3.2) were not true, there would be a minimal $s>t_{0}$ such that $y_{s}^{2}=L s$ and $y_{t}^{2} \geq L t$ on $[0, s]$. It follows from (2.7) that

$$
\partial_{t} y_{t}^{2}=\frac{4 y_{t}^{2}}{x_{t}^{2}+y_{t}^{2}} \geq \frac{4 L t}{M^{2} t+L t}=\frac{4 L}{M^{2}+L} \geq L
$$

for all $t_{0} \leq t \leq s$, which implies $y_{s}^{2}-y_{t_{0}}^{2} \geq L\left(s-t_{0}\right)$. This contradicts the fact

$$
y_{s}^{2}-y_{t_{0}}^{2}=L s-y_{t_{0}}^{2}<L\left(s-t_{0}\right) .
$$

If $\sigma<2$, then the assumption $\left|x_{t}\right|<M \sqrt{t}$ of the Lemma is satisfied with $M=\sigma$ by Lemma 2.1 and arbitrarily small $C$, and Theorem 3.1 follows easily. The reader who is only interested in a short proof of Theorem 1.1 for small Hölder-1/2 norm may thus skip ahead to Corollary 3.5. To deal with the case $2 \leq \sigma<4$, we will show that the trivial bound $\left|x_{t}\right| \leq \sigma \sqrt{t}$ can be improved to an estimate $\left|x_{t}\right|<M \sqrt{t}$ for some $M<2$ and $t$ large enough, if we assume that $z_{t}$ stays outside a cone. As a first step, we will show:

Lemma 3.3. Let $K$ and $M$ be finite positive constants. If

$$
K y_{t} \leq x_{t} \leq M \sqrt{t} \quad \text { for all } t \in\left[t_{0}, T\right]
$$

then

$$
x_{t} \leq\left(\sigma-\frac{4 K^{2}}{K^{2}+1} \frac{1}{M}\right) \sqrt{t}+C \quad \text { for all } t \in\left[t_{0}, T\right],
$$

where $C=\left(M+4 K^{2} /\left(M\left(K^{2}+1\right)\right)\right) \sqrt{t_{0}}$.

Proof. It follows from the differential equation (2.6) for $x_{t}+\xi_{t}$ that

$$
x_{t}+\xi_{t}-x_{t_{0}}-\xi_{t_{0}}=\int_{t_{0}}^{t} \frac{-2 x_{s}}{x_{s}^{2}+y_{s}^{2}} d s=\int_{t_{0}}^{t} \frac{-2\left(\frac{x_{s}}{y_{s}}\right)^{2}}{\left(\frac{x_{s}}{y_{s}}\right)^{2}+1} \frac{1}{x_{s}} d s \leq \frac{-2 K^{2}}{K^{2}+1} \int_{t_{0}}^{t} \frac{1}{x_{s}} d s,
$$

so that

$$
\begin{aligned}
x_{t} & \leq x_{t_{0}}-\frac{2 K^{2}}{K^{2}+1} \int_{t_{0}}^{t} \frac{1}{x_{s}} d s+\sigma \sqrt{t} \leq M \sqrt{t_{0}}-\frac{2 K^{2}}{K^{2}+1} \int_{t_{0}}^{t} \frac{1}{M \sqrt{s}} d s+\sigma \sqrt{t} \\
& =\left(\sigma-\frac{4 K^{2}}{\left(K^{2}+1\right) M}\right) \sqrt{t}+\left(M+\frac{4 K^{2}}{\left(K^{2}+1\right) M}\right) \sqrt{t_{0}} .
\end{aligned}
$$


Lemma 3.4. For every $\sigma<4$ and $\sigma^{\prime}>\sigma / 2$ there are $K>0$ and $C>0$ such that, if $x_{0}=K y_{0}$ and if $x_{t} \geq K y_{t}$ for all $t \geq 0$, then $\left|x_{t}\right| \leq \sigma^{\prime} \sqrt{t}$ for all $t \geq C y_{0}^{2}$.

Proof. Let $M_{0}=\sigma$, and $K=\sigma / \sqrt{16-\sigma^{2}}$. Recursively define

$$
M_{n+1}=\sigma-\frac{4 K^{2}}{\left(K^{2}+1\right) M_{n}}
$$

and notice that $M_{n} \rightarrow \sigma / 2$ as $n \rightarrow \infty$. Hence there is $N$ such that $M_{N}<\sigma^{\prime}$. Because $x_{t} \leq x_{0}+\sigma \sqrt{t}$, for every $M_{0}^{\prime}>\sigma$ there is $C_{0}$ such that $x_{t} \leq M_{0}^{\prime} \sqrt{t}$ for all $t \in\left[C_{0} y_{0}^{2}, T\right]$. It follows from Lemma 3.3 that for every $M_{1}^{\prime}>M_{1}$ there is $C_{1}$ such that $x_{t} \leq M_{1}^{\prime} \sqrt{t}$ for all $t \in\left[C_{1} y_{0}^{2}, T\right]$. Similarly, by continuity and $N$ applications of Lemma 3.3, for every $M_{N}^{\prime}>M_{N}$ there is $C_{N}$ such that $x_{t} \leq M_{N}^{\prime} \sqrt{t}$ for all $t \in\left[C_{N} y_{0}^{2}, T\right]$. The lemma follows by choosing $M_{N}^{\prime}=\sigma^{\prime}$ and setting $C=C_{N}$.

We are now ready to give the:

Proof of Theorem 3.1. If $\sigma<2$, we simply apply Lemma 3.2 with arbitrarily small $C$ and find that

$$
\frac{\left|x_{t}\right|}{y_{t}} \leq \frac{\sigma \sqrt{t}}{L \sqrt{t}}=\frac{\sigma}{\sqrt{4-\sigma^{2}}}
$$

for all $t$ so that we can take $c_{\sigma}=\sigma / \sqrt{4-\sigma^{2}}$. In general, fix $\sigma^{\prime} \in(\sigma / 2,2)$ and let $K$ and $C$ be the constants of Lemma 3.4. Since $x_{0}=0$, the points $z_{t}$ are in the cone $A_{K}$ for all small $t$ (see Figure 1). If for some $t$, the point $z_{t}$ is outside $A_{K}$, then we can find an interval $\left[t_{1}, t_{2}\right]$ containing $t$ so that

$$
\left|x_{t_{1}}\right|=K y_{t_{1}} \text { and }\left|x_{s}\right| \geq K y_{s} \text { for all } t_{1} \leq s \leq t_{2},
$$

and without loss of generality we may assume $x_{s}>0$ on $\left[t_{1}, t_{2}\right]$. Replacing $\xi$ by $\tilde{\xi}(\cdot)=\xi\left(\cdot+t_{1}\right)-\xi\left(t_{1}\right)$ on $\left[0, t_{2}-t_{1}\right]$, we are now in the situation where $x_{0}=K y_{0}$ and $x_{t} \geq K y_{t}$ for all $t \in[0, T]$ (where $T=t_{2}-t_{1}$ ). By Lemma 3.4, we can apply Lemma 3.2 and obtain

$$
\frac{x_{t}}{y_{t}} \leq \frac{\sigma^{\prime} \sqrt{t}}{L \sqrt{t}}=\sigma^{\prime} \max \left(C, 1 /\left(4-\sigma^{\prime 2}\right)\right)
$$

for $t \geq C y_{0}^{2}$, whereas

$$
\frac{x_{t}}{y_{t}} \leq \frac{x_{0}+\sigma \sqrt{t}}{y_{0}} \leq K+\sigma \sqrt{C}
$$

for $t \leq C y_{0}^{2}$. It follows that $z_{t}$ never leaves the cone $A_{c}$ where $c=\max \left(\sigma^{\prime} C, \sigma^{\prime} /(4-\right.$ $\left.\left.\sigma^{\prime 2}\right), \bar{K}+\sigma \sqrt{C}\right)$.

Finally, the estimate for $y_{t}$ follows from $\left|x_{t}\right| \leq c y_{t}$ and

$$
\partial_{t} y_{t}^{2}=\frac{4 y_{t}^{2}}{x_{t}^{2}+y_{t}^{2}}
$$

A simple consequence of Theorem 3.1 is the Hölder continuity in bounded subsets of the upper half plane of the solutions $f_{t}$ to the upward Loewner equation $(2.3)$ with driving functions satisfying $\sigma=\|\xi\|_{1 / 2}<4$ : 


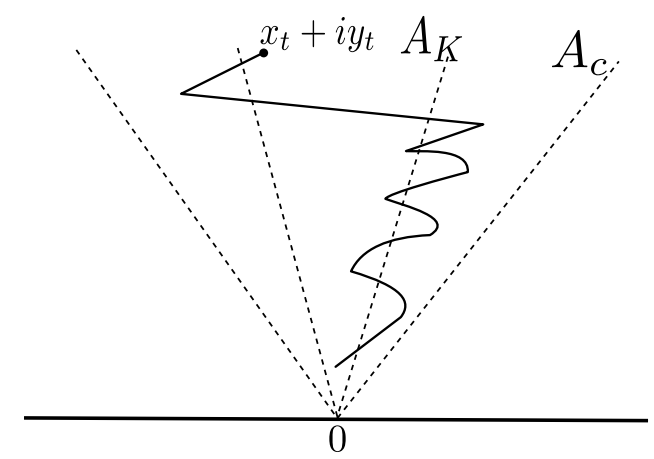

Figure 1: A trajectory of $x_{t}+i y_{t}$. It never leaves the cone $A_{c}$ once outside $A_{K}$.

Corollary 3.5. If $\sigma=\|\xi\|_{1 / 2}<4$, then

$$
\left|f_{t}^{\prime}\left(\xi_{0}+i y\right)\right| \leq\left(4 t+y^{2}\right)^{(1-\alpha) / 2} y^{\alpha-1}
$$

for every $y>0$ and $t \in[0, T]$, where $\alpha$ is a constant in $(0,1]$ depending on $\sigma$ only.

Proof. By (2.7) and (2.8), Theorem 3.1 implies that

$$
\left|f_{t}^{\prime}\left(\xi_{0}+i y\right)\right| \leq \exp \left(\int_{0}^{t} \frac{c^{2}-1}{c^{2}+1} \frac{2 d s}{x_{s}^{2}+y_{s}^{2}}\right)=\left(\frac{y_{t}}{y}\right)^{\frac{c^{2}-1}{c^{2}+1}} \leq\left(4 t+y^{2}\right)^{(1-\alpha) / 2} y^{\alpha-1},
$$

where $c=c_{\sigma}$ and $\alpha=\min \left\{1-\frac{c^{2}-1}{c^{2}+1}, 1\right\} \in(0,1]$.

Remark 3.6. The proof of Theorem 3.1 can easily be modified to give the following statement: for every $0<c_{1}<c_{2}$, there is $\sigma_{0}$ such that, if $z_{0} \in A_{c_{1}}$ and $\sigma \leq \sigma_{0}$, then $z_{t} \in A_{c_{2}}$ for all $t$. Then (3.1) holds with $c_{\sigma}$ replaced by $c_{2}$.

Corollary 3.7. There is a constant $\sigma_{0}$ such that the following is true: if $\|\xi\|_{1 / 2} \leq$ $\sigma_{0}$, if $0 \leq c \leq 1$ and $z$ is in the cone $A_{c}\left(\xi_{0}\right)$, and if

$$
\int_{0}^{T} \frac{M_{0, s}^{\xi}}{s^{3 / 2}} d s<\infty
$$

then

$$
\left|\arg f_{T}^{\prime}(z)\right| \leq 8 c+4 \int_{0}^{T} \frac{M_{0, s}^{\xi}}{s^{3 / 2}} d s
$$

Proof. Let $\sigma_{0}$ be the constant from Remark 3.6 with $c_{1}=1$ and $c_{2}=\sqrt{3}$. Then if $z_{0} \in A_{c}$ and $c \leq 1$, we have $z_{t} \in A_{c_{2}}$ and $y_{t} \geq \sqrt{y_{0}^{2}+t}$ for all $t$ by (3.1). By (2.9) and Lemma 2.1,

$$
\begin{aligned}
\left|\arg f_{T}^{\prime}(z)\right| & \leq 4 \int_{0}^{T} \frac{\left|x_{s}\right|}{y_{s}^{3}} d s \leq 4 \int_{0}^{T} \frac{c y_{0}+M_{0, s}^{\xi}}{\left(y_{0}^{2}+s\right)^{3 / 2}} d s \\
& =8 c y_{0}\left(\frac{1}{y_{0}}-\frac{1}{\sqrt{y_{0}^{2}+T}}\right)+4 \int_{0}^{T} \frac{M_{0, s}^{\xi}}{s^{3 / 2}} d s \leq 8 c+4 \int_{0}^{T} \frac{M_{0, s}^{\xi}}{s^{3 / 2}} d s .
\end{aligned}
$$




\section{The proofs of Theorems 1.1 and 1.2}

Throughout this section, we maintain our notation $\sigma=\|\lambda\|_{1 / 2}$, and denote by $\alpha=\alpha_{\sigma}$ the constant of Corollary 3.5. As explained in Section 2, in order to show that the Loewner equation generates a curve it suffices to show that

$$
v(t, \varepsilon):=\int_{0}^{\varepsilon}\left|\left(g_{t}^{-1}\right)^{\prime}\left(\lambda_{t}+i y\right)\right| d y
$$

goes to zero as $\varepsilon \rightarrow 0$, uniformly in $t \in[0, T]$. In our setting, this follows easily from Corollary 3.5.

Lemma 4.1. Suppose that $\lambda:[0, T] \rightarrow \mathbb{R}$ is Hölder continuous with $\sigma<4$ and $\left(g_{t}\right)$ is the solution to (2.1). Then for every $\varepsilon>0$ and $0 \leq t \leq T$,

$$
\int_{0}^{\varepsilon}\left|\left(g_{t}^{-1}\right)^{\prime}\left(\lambda_{t}+i y\right)\right| d y \leq \frac{\left(4 t+\varepsilon^{2}\right)^{(1-\alpha) / 2}}{\alpha} \varepsilon^{\alpha} .
$$

Proof. Fix $0 \leq t \leq T$ and $\varepsilon>0$. Let $\xi_{s}=\lambda_{t-s}$ for $0 \leq s \leq t$. Let $\left(f_{s}\right)_{0 \leq s \leq t}$ be the solution to (2.3) with the driving function $\xi$, so that $g_{t}^{-1}=f_{t}$. Hence by Corollary 3.5,

$$
\left|\left(g_{t}^{-1}\right)^{\prime}\left(\lambda_{t}+i y\right)\right|=\left|f_{t}^{\prime}\left(\xi_{0}+i y\right)\right| \leq\left(4 t+y^{2}\right)^{(1-\alpha) / 2} y^{\alpha-1},
$$

and the lemma follows by integration.

Remark 4.2. By Proposition 3.9 of [2], we get a quantitative estimate for the modulus of continuity of the trace $\gamma_{t}:=\lim _{y \rightarrow 0^{+}} g_{t}^{-1}\left(\lambda_{t}+i y\right)$, namely $\gamma$ is Hölder continuous with exponent $\alpha / 2$.

To complete the proof of Theorem 1.1, it only remains to show that $\gamma$ is a simple curve and satisfies the Ahlfors geometric characterization of quasiconformal arcs

$$
\left|\gamma_{t}-\gamma_{s}\right| \leq M\left|\gamma_{t}-\gamma_{r}\right|
$$

for some constant $M=M_{\gamma}$ and all $0 \leq r \leq s \leq t \leq T$. The key idea is to use the Gehring-Hayman inequality, see page 72 in [9], which says that among all curves in a simply connected plane domain with two fixed end points, the hyperbolic geodesic minimizes the euclidean length, up to a universal multiplicative constant.

Lemma 4.3. If $\sigma<4$, then $\gamma$ is a simple curve that stays inside the cone $A_{c_{\sigma}}\left(\lambda_{0}\right)$ and satisfies (4.2).

Proof. Again consider the upward Loewner equation (2.3) with the driving function $\xi(s)=\lambda(t-s)$ for $s \in[0, t]$, for fixed $t \in[0, T]$. It follows from Theorem 3.1 that, for $z=\xi_{0}+i \varepsilon=\lambda_{t}+i \varepsilon$,

$$
\left|x_{t}\right| \leq c_{\sigma} y_{t}
$$

and

$$
\sqrt{\frac{4}{1+c_{\sigma}^{2}} t+\varepsilon^{2}} \leq y_{t}^{2} \leq \sqrt{4 t+\varepsilon^{2}} .
$$


Since $\gamma_{t}=\lim _{\varepsilon \rightarrow 0^{+}}\left(g_{t}^{-1}\right)\left(\lambda_{t}+i \varepsilon\right)=\lim _{\varepsilon \rightarrow 0^{+}}\left(x_{t}+i y_{t}+\xi_{t}\right)$, it follows that

$$
\frac{2 \sqrt{t}}{\sqrt{1+c_{\sigma}^{2}}} \leq \operatorname{Im} \gamma_{t} \leq 2 \sqrt{t}
$$

and

$$
\left|\operatorname{Re} \gamma_{t}-\lambda_{0}\right| \leq c_{\sigma} \operatorname{Im} \gamma_{t} \leq 2 c_{\sigma} \sqrt{t}
$$

This implies that the curve $\gamma$ is contained in the cone $A_{c_{\sigma}}\left(\lambda_{0}\right)$ and meets the real line non-tangentially. It also implies that $\gamma(0, T] \cap \mathbb{R}=\emptyset$, which easily implies that $\gamma$ is simple (Lemma 4.34 in [4]): just notice that, if $\gamma_{t}=\gamma_{t^{\prime}}$ for some $t<t^{\prime}$, then $g_{t}(\gamma(t, T])$ intersects the real line at $\lambda_{t}$, but that the curve $g_{t}(\gamma[t, T])$ has driving function $\tilde{\lambda}_{t}=\lambda_{t+t^{\prime}}$ so that $g_{t}(\gamma(t, T]) \cap \mathbb{R}=\emptyset$ by the above.

To prove (4.2), fix $0 \leq r \leq s \leq t \leq T$, denote $\gamma_{r}, \gamma_{s}, \gamma_{t}$ by $u, v, w$, and their images under $g_{r}$ by $u^{\prime}, v^{\prime}, w^{\prime}$. We may assume that the line segment $(u, w)$ is contained in $H_{r}=\mathbb{H} \backslash \gamma[0, r]$ (else replace $u$ by the point $\hat{u}$ that is closest to $w$ on $(u, w) \cap \gamma[0, r]$, and replace $r$ by $\left.\hat{r}=\gamma^{-1}(\hat{u})\right)$. By (4.3),

$$
\operatorname{Im} v^{\prime} \leq 2 \sqrt{s-r} \leq 2 \sqrt{t-r} \leq \sqrt{1+c_{\sigma}^{2}} \operatorname{Im} w^{\prime}
$$

so that the hyperbolic geodesic geo ${ }_{\mathbb{H}}\left(u^{\prime}, v^{\prime}\right)$ from $u^{\prime}$ to $v^{\prime}$ in $\mathbb{H}$ is within bounded hyperbolic distance from geo $_{\mathbb{H}}\left(u^{\prime}, w^{\prime}\right)$. In particular, there is a point $z^{\prime}=g_{r}(z)$ on geo $_{\mathbb{H}}\left(u^{\prime}, w^{\prime}\right)$ of bounded hyperbolic distance from $v^{\prime}$ (where all bounds depend on $c_{\sigma}$ only). Denoting $\ell$ the euclidean length, it follows from the Koebe distortion theorem that

$$
|v-w| \leq|v-z|+|z-w| \leq C \operatorname{dist}\left(z, \partial H_{r}\right)+\ell\left(\operatorname{geo}_{H_{r}}(z, w)\right) \leq C \ell\left(\operatorname{geo}_{H_{r}}(u, w)\right) .
$$

Since the line segment $(u, w)$ is contained in $H_{r}$, the Gehring-Hayman inequality implies $\ell\left(\operatorname{geo}_{H_{r}}(u, w)\right) \leq C^{\prime}|u-w|$ and (4.2) follows. This finishes the proof of Lemma 4.3 and of Theorem 1.1.

Proof of Theorem 1.2. Since we did not assume a priori that $\lambda$ generates a curve, we first observe that

$$
\|\lambda\|_{1 / 2} \leq 3 C_{0}
$$

Indeed, since $\left|\lambda_{t_{2}}-\lambda_{t_{1}}\right| \leq 2 N_{s, t_{2}}^{\lambda}$ for $0<s \leq t_{1}<t_{2} \leq T$, it is not hard to see that $\lambda$ has a finite Hölder-1/2 norm on every interval $\left[t_{1}, t_{2}\right]$ inside $(0, T]$. Next, for $t_{1} \leq s \leq r \leq t \leq t_{2}$ we have $\left|\lambda_{t}-\lambda_{s}\right| \leq N_{r, t}^{\lambda}+\|\lambda\|_{1 / 2} \sqrt{r-s}$. Integrating both sides of this inequality from $s$ to $t$ with respect to $r$, dividing by $(t-s)^{3 / 2}$ and estimating the integral involving $N^{\lambda}$ by $C_{0}$, (4.5) easily follows by choosing $s$ and $t$ appropriately.

If $C_{0}<4 / 3$, Theorem 1.1 applies and $\lambda$ generates a curve $\gamma$. We will show that, if $C_{0}$ is small enough, then for every pair of points $\gamma_{t_{1}}, \gamma_{t_{2}}$ on $\gamma$ with $0 \leq t_{1}<t_{2} \leq T$ we have

$$
\left|\arg \left(\gamma_{t_{2}}-\gamma_{t_{1}}\right)-\frac{\pi}{2}\right| \leq C<\frac{\pi}{2}
$$


where $C$ depends on $C_{0}$ and $\sigma=\|\lambda\|_{1 / 2}$ only, and where $\arg (z) \in(-\pi, \pi]$ is the principal argument of $z$. This implies that $\gamma$ grows vertically and is the graph of a Lipschitz function.

Let $\tilde{\gamma}$ be the image of $\gamma$ under the map $g_{t_{1}}$. This is the curve generated by the driving function $\tilde{\lambda}_{t}=\lambda_{t+t_{1}}, t \in\left[0, T-t_{1}\right]$, see the figure below.

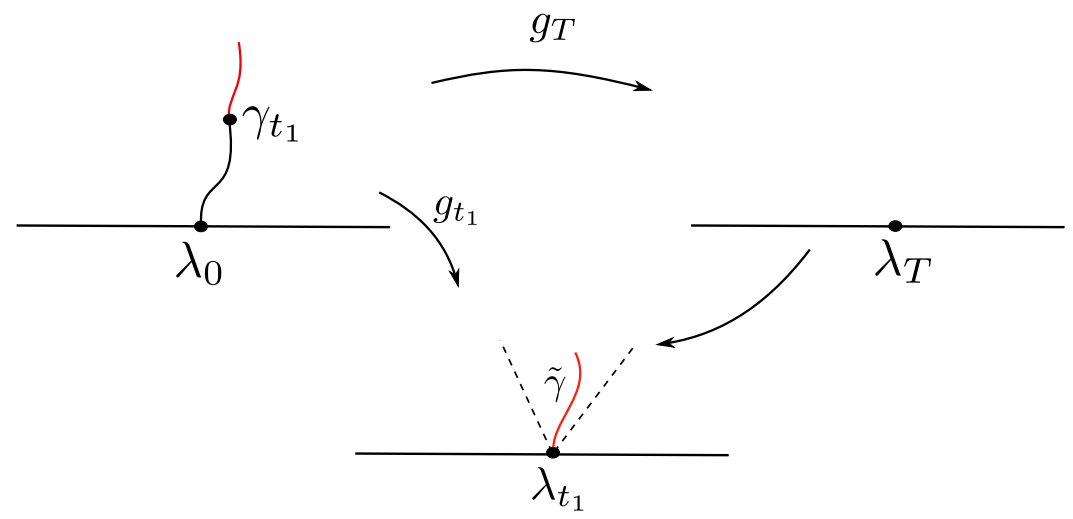

Figure 2: The curve $\tilde{\gamma}$ is inside the cone $A_{c}\left(\lambda_{t_{1}}\right)$.

By Lemma 4.3 the curve $\tilde{\gamma}$ is in the cone $A_{c}\left(\lambda_{t_{1}}\right)$, where $c=c_{\sigma}$ is defined in Section 3. With $w=g_{t_{1}}\left(\gamma_{t_{2}}\right)-\lambda_{t_{1}}$ we therefore have

$$
\begin{aligned}
\left|\arg \left(\gamma_{t_{2}}-\gamma_{t_{1}}\right)-\frac{\pi}{2}\right| & =\left|\arg \left(w \int_{0}^{1}\left(g_{t_{1}}^{-1}\right)^{\prime}\left(\lambda_{t_{1}}+s w\right) d s\right)-\frac{\pi}{2}\right| \\
& \leq \arctan c+\sup _{z \in A_{c}\left(\lambda_{t_{1}}\right)}\left|\arg \left(g_{t_{1}}^{-1}\right)^{\prime}(z)\right| .
\end{aligned}
$$

Applying Corollary 3.7 to the driving function $\xi_{t}=\lambda_{t_{1}-t}$ with $t \in\left[0, t_{1}\right]$, assuming $C_{0}$ is small enough such that $3 C_{0}<\sigma_{0}$ and $c_{3 C_{0}} \leq 1$, we get

$$
\sup _{z \in A_{c}\left(\lambda_{t_{1}}\right)}\left|\arg \left(g_{t_{1}}^{-1}\right)^{\prime}(z)\right| \leq 8 c+4 \int_{0}^{t_{1}} \frac{M_{0, s}^{\xi}}{s^{3 / 2}} d s=8 c+4 \int_{0}^{t_{1}} \frac{N_{s, t_{1}}^{\lambda}}{\left(t_{1}-s\right)^{3 / 2}} d s .
$$

Thus

$$
\left|\arg \left(\gamma_{t_{2}}-\gamma_{t_{1}}\right)-\frac{\pi}{2}\right| \leq \arctan c+8 c+4 C_{0} .
$$

If $C_{0} \rightarrow 0$, then $\sigma \rightarrow 0$ by (4.5) and therefore $c \rightarrow 0$ by Theorem 3.1. Thus (4.6) follows if $C_{0}$ is sufficiently small, and the theorem is proved.

\section{References}

[1] Astala, K., Jones, P., Kupiainen, A. And Saksman, E.: Random conformal weldings. Acta Math. 207 (2011), no. 2, 203-254. 
[2] Johansson Viklund, F. and Lawler, G. F.: Optimal Hölder exponent for the SLE path. Duke Math. J. 159 (2011), no. 3, 351-383.

[3] Kager, W., Nienhuis, B. And Kadanoff, L. P.: Exact solutions for Loewner evolutions. J. Statist. Phys. 115 (2004), no. 3-4, 805-822.

[4] LaWler, G. F.: Conformally invariant processes in the plane. Mathematical Surveys and Monographs, American Mathematical Society, Providence, RI, 2005.

[5] Lawler, G.F.: Schramm-Loewner evolution. In Statistical mechanics, 231-295. IAS/Park City Math. Ser. 16, Amer. Math. Soc., Providence, RI, 2009.

[6] Lind, J. R.: A sharp condition for the Loewner equation to generate slits. Ann. Acad. Sci. Fenn. Math. 30 (2005), no. 1, 143-158.

[7] LÖwner, K.: Untersuchungen über schlichte konforme Abbildungen des Einheitskreises I. Math. Ann. 89 (1923), no. 1-2, 103-121.

[8] Marshall, D. E. And Rohde, S.: The Loewner differential equation and slit mappings. J. Amer. Math. Soc. 18 (2005), no. 4, 763-778.

[9] Pommerenke, C.: Boundary behaviour of conformal maps. Grundlehren der Mathematischen Wissenschaften 299, Springer-Verlag, Berlin, 1992.

[10] Rohde, S. And Schramm, O.: Basic properties of SLE. Ann. Math. (2) 161 (2005), no. 2, 883-924.

[11] Schramm, O.: Scaling limits of loop-erased random walks and uniform spanning trees. Israel J. Math. 118 (2000), 221-288.

[12] Sheffield, S. : Conformal weldings of random surfaces: SLE and the quantum gravity zipper. Ann. Probab. 44 (2016), no. 5, 3474-3545.

Received March 3, 2016.

Steffen Rohde: Department of Mathematics, University of Washington, Box 354350, Seattle, 98195 WA, USA.

E-mail: rohde@math.washington.edu

HuY Tran: Institut für Mathematik, Technische Univesität Berlin, Strasse des 17. Juni 136, 10623 Berlin, Germany.

E-mail: tran@math.tu-berlin.de

Michel Zinsmeister: Université d'Orléans, UFR Sciences, Bâtiment de Mathématiques, Rue de Chartres, B. P. 6759, 45067 Orléans cedex 2, France.

E-mail: zins@univ-orleans.fr

Steffen Rohde's research is supported by NSF grant DMS-1068105. The project was done while the second author was a graduate student at University of Washington, Seattle. He thanks the institute for hospitality and generosity. The authors would like to thank anonymous referees for helpful comments. 